\title{
ON THE PROBLEM OF THE MINIMUM SUM OF THE DISTANCES OF A POINT FROM GIVEN POINTS.*
}

BY PROF. V. SCHIJGEL, OF HAGEN I. W.

CONTENTS.

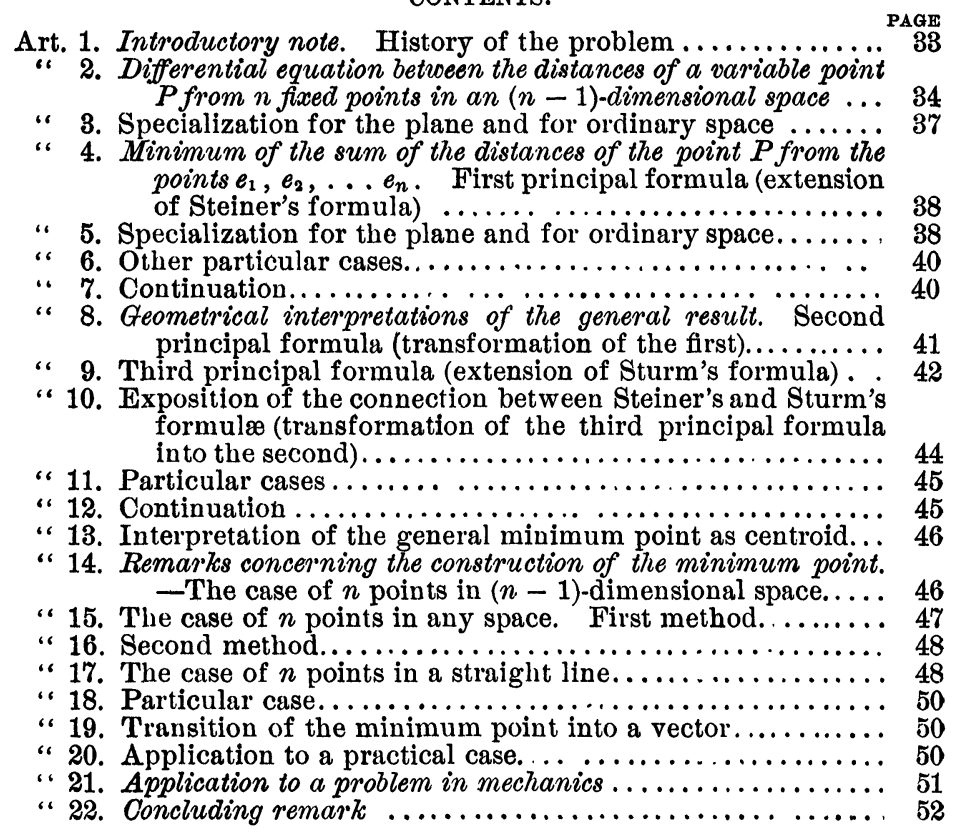

1. Introductory Note.-Although repeatedly treated before, $\dagger$ the problem of determining a point such that the sum of its distances from a number of given points shall be a minimum still offers room for discussion in two respects.

First, the question as to the best method of investigation cannot yet be regarded as settled. The ordinary method of the differential calculus not only leads to unnecessary complications owing to the use of co-ordinates not essentially connected with the problem, but also necessitates the exclusion of negative distances, a serious difficulty that does not seem to have been

* Presented to the American Mathematrcal Socretr, at its summer meeting, Aug. 15, 1894 . Translated by A. Z.

+ See, for instance, the references to the literature of the problem given by SToRM in Crelle's Journal für Mathematik, vol. 97, p. 51. 
overcome.* The methods of elementary geometry, on the other hand, always comprise only a comparatively small class of problems, and even the simplest solutions, like those for the sum of the distances from three or four points, give decidedly the impression of being nothing but special artifices. It is to the researches of Sturm that we owe the important result that all these particular solutions can be regarded as special cases of a general method applicable to $n$ points. Nevertheless, there seems to be but little prospect of applying these purely geometrical methods successfully to those generalized problems that are mentioned by Steiner in the brief notice of his paper read before the Berlin Academy. $\uparrow$ Indeed, the character of these generalizations which relate to sums, not of mere distances, but of multiples of powers of the distances, would seem to point in the direction of analytical methods. The single result for this kind of problems given by Steiner himself $\ddagger$ bears so openly the mark of an analytical origin that an analytical investigation of the generalized problem appears to offer the best chances of success in uncovering the "easy geometrical considerations" that sufficed Steiner, as is implied in the notice of his paper, to solve the most general case of the problem.

Secondly, our problem lends itself to a series of generalizations which do not appear as yet to have been considered.

In what follows the problem is treated by means of the simplest methods of Grassmann's geometrical calculus (Ausdehnungslehre).

2. Differential equation between the distances of a variable point $P$ from $n$ fixed points in an $(n-1)$-dimensional space. Let $P$ be a variable point derived from $n$ fixed points $e_{1}$, $e_{2}, \ldots e_{n}$ by means of the numbers $\alpha_{1}, \alpha_{2}, \ldots \alpha_{n}$, so that

$$
\begin{gathered}
P=\alpha_{1} e_{1}+\alpha_{2} e_{2}+\ldots+\alpha_{n} e_{n} ; \quad \alpha_{1}+\alpha_{2}+\ldots+\alpha_{n}=1 ; \\
\left(e_{1} e_{2} \ldots e_{n}\right)=1 .
\end{gathered}
$$

Then the outer product $\left(e_{1} e_{2} \ldots e_{n}\right)$ represents the $(n-1)$ !fold volume of the $(n-1)$-dimensional $n$-gon formed by the points $e_{1}, e_{2}, \ldots e_{n}$, i.e., of the $n$th term in the series of configurations beginning with the terms: point, rectilinear segment, triangle, tetrahedron, pentahedroid (Fünfzell), etc. The coefficients $\alpha_{1}, \alpha_{2}, \ldots \alpha_{n}$ represent the $(n-1)$ !-fold volumes of the $(n-1)$-dimensional $n$-gons $P e_{2} e_{3} \ldots e_{n}, P e_{1} e_{3} \ldots e_{n}, \ldots$

* See STURM, l. c.

$\dagger$ Monatsberichte der Akademie der Wissenschaften, Berlin, 1837, p 144 ; STwiner, Gesammelte Werke, vol. II. p. 95.

$\ddagger$ Crelle's Journal für Mathematik, vol. 13, p. 362 ; STEINER, Gesam Werke, vol. II. p. 17 . 
$P e_{1} e_{2} \ldots e_{n-1}$. For we find from (1) by outer multiplication, say by $\left(e_{2} e_{3} \ldots e_{n}\right)$,

$$
\left(P e_{2} e_{3} \ldots e_{n}\right)=\alpha_{1}\left(e_{1} e_{2} \ldots e_{n}\right)=\alpha_{1} .
$$

Now $\left(P e_{1}\right)=\alpha_{2}\left(e_{2} e_{1}\right)+\alpha_{3}\left(e_{3} e_{1}\right)+\ldots+\alpha_{n}\left(e_{n} e_{1}\right) ;$ and forming the inner square:

$$
\left(P e_{1}\right)^{2}=\Sigma \alpha_{r}^{2}\left(e_{r} e_{1}\right)^{2}+\Sigma \alpha_{r} \alpha_{s}\left[e_{r} e_{1} \mid e_{s} e_{1}\right] ; r \lesseqgtr s ; r, s=2,3, \ldots n \text {. }
$$

On the other hand, denoting by $p_{1}, a_{1 r}, a_{1 s}$ the numerical values of the rotors or point-vectors (Linienteile) $\left(P e_{1}\right),\left(e_{r} e_{1}\right)$, $\left(e_{s} e_{1}\right)$, we have, with the same conditions for $r$ and $s$,

$$
p_{1}^{2}=\Sigma \alpha_{r}^{2} a_{1 r}^{2}+\sum \alpha_{r} \alpha_{s} a_{1 r} \alpha_{1 s} \cos \phi_{r 1 s},
$$

where $\phi_{r 1 s}$ is the angle between the edges $a_{1 r}$ and $a_{1 s}$. Replacing the expression $a_{1 r} a_{1 s} \cos \phi_{r 1 s}$ by $\frac{1}{2}\left(a_{1 r}{ }^{2}+a_{1 s}{ }^{2}-a_{r s}{ }^{2}\right)$, we find

$$
p_{1}^{2}=\Sigma \alpha_{r}^{2} a_{1 r}^{2}+\frac{1}{2} \Sigma \alpha_{r} \alpha_{s}\left(\alpha_{1 r}^{2}+a_{1 s}^{2}-\alpha_{r s}^{2}\right),
$$

or, writing separately the terms in which $r, s=n$,

$$
\begin{aligned}
& p_{1}^{2}=\alpha_{n}^{2} a_{1 n}^{2}+\Sigma \alpha_{r}^{2} a_{1 r}^{2}+\Sigma \alpha_{r} \alpha_{n}\left(a_{1 r}^{2}+a_{1 n}^{2}-a_{r n}^{2}\right) \\
& +\frac{1}{2} \sum \alpha_{r} \alpha_{s}\left(\alpha_{1 r}^{2}+a_{1 s}^{2}-\alpha_{r s}^{2}\right) ; r, s=2,3, \ldots(n-1) \text {. }
\end{aligned}
$$

According to equation (1), the position of $P$ is determined by the co-ordinates $\alpha_{1}, \alpha_{2}, \ldots \alpha_{n}$, of which $(n-1)$, say $\alpha_{1}$, $\alpha_{2}, \ldots \alpha_{n-1}$, can be regarded as independent. As, by (1),

we have

$$
\alpha_{n}=1-\alpha_{1}-\alpha_{2}-\ldots-\alpha_{n-1},
$$

$$
\frac{d \alpha_{n}}{d \alpha_{1}}=\frac{d \alpha_{n}}{d \alpha_{2}}=\ldots=\frac{d \alpha_{n}}{d \alpha_{n-1}}=-1
$$

Hence (2) gives:

$$
\begin{gathered}
\frac{d\left(p_{1}^{2}\right)}{d \alpha_{1}}=-2 \alpha_{n} a_{1 n}{ }^{2}-\Sigma \alpha_{r}\left(a_{1 r}{ }^{2}+{a_{1 n}}^{2}-{a_{r n}}^{2}\right) ; \\
r=2,3, \ldots(n-1) .
\end{gathered}
$$

Let $k$ denote any one of the subscripts $2,3, \ldots(n-1)$; then we have by analogy with (2)

$$
\begin{gathered}
p_{k}^{2}=\Sigma \alpha_{r}^{2} a_{k r}^{2}+\frac{1}{2} \Sigma \alpha_{r} \alpha_{s}\left(a_{k r}^{2}+a_{k s}^{2}-a_{r s}^{2}\right) ; \\
r \gtreqless s ; r, s=1,2, \ldots(k-1),(k+1), \ldots n .
\end{gathered}
$$


or, writing separately the terms in which $r, s=1, n$,

$$
\begin{aligned}
& p_{k}^{2}=\alpha_{1}^{2} a_{k 1}^{2}+\alpha_{n}^{2} a_{k n}^{2}+\Sigma \alpha_{r}^{2} \alpha_{k r}^{2}+\alpha_{1} \alpha_{n}\left(a_{k 1}^{2}+a_{k n}^{2}-a_{1 n}^{2}\right) \\
& +\sum \alpha_{r} \alpha_{1}\left(a_{k r}^{2}+a_{k 1}^{2}-a_{r 1}^{2}\right)+\sum \alpha_{r} \alpha_{n}\left(a_{k r}^{2}+a_{k n}^{2}-a_{r n}^{2}\right) \\
& +\frac{1}{2} \Sigma \alpha_{r} \alpha_{s}\left(a_{k r}^{2}+a_{k s}^{2}-a_{r s}^{2}\right) \text {; } \\
& r, s=2,3, \ldots(k-1),(k+1), \ldots(n-1) .
\end{aligned}
$$

Hence

$$
\begin{aligned}
\frac{d\left(p_{k}^{2}\right)}{d \alpha_{1}}= & \alpha_{n}\left(a_{k 1}^{2}-a_{n 1}^{2}-a_{k n}{ }^{2}\right)+\alpha_{1}\left(a_{k 1}^{2}+a_{n 1}{ }^{2}-a_{k n}{ }^{2}\right) \\
& +\sum \alpha_{r}\left(a_{k 1}{ }^{2}-a_{r 1}^{2}+a_{r n}{ }^{2}-a_{k n}{ }^{2}\right) \\
& \quad r=2,3, \ldots(k-1),(k+1), \ldots(n-1) .
\end{aligned}
$$

Interchanging the subscripts 1 and $n$ in (3), we find by differentiation

$\frac{d\left(p_{n}^{2}\right)}{d \alpha_{1}}=2 \alpha_{1} \alpha_{n 1}^{2}+\Sigma \alpha_{r}\left(a_{n 1}^{2}+a_{n r}{ }^{2}-a_{1 r}^{2}\right) ; r=2,3, \ldots(n-1)$

Let us now substitute in $(7)$ the values $2,3, \ldots(n-1)$ successively for $k$ and add all the resulting equations, together with the equations (4) and (8), after multiplying them respectively by $\alpha_{k}, \alpha_{1}, \alpha_{n}$; in the resulting equation,

$$
\begin{aligned}
& \alpha_{1} \frac{d\left(p_{1}^{2}\right)}{d \alpha_{1}}+\Sigma \alpha_{k} \frac{d\left(p_{k}^{2}\right)}{d \alpha_{1}}+\alpha_{n} \frac{d\left(p_{n}^{2}\right)}{d \alpha_{1}}=-2 \alpha_{n} \alpha_{1} \alpha_{1 n}^{2} \\
& -\alpha_{1} \Sigma \alpha_{r}\left(a_{1 n}{ }^{2}+a_{1 n}^{2}-a_{r n}^{2}\right)+\alpha_{n} \Sigma \alpha_{k}\left(a_{k 1}{ }^{2}-a_{n 1}^{2}-a_{k n}^{2}\right) \\
& +\alpha_{1} \Sigma \alpha_{k}\left(a_{k 1}^{2}+a_{n 1}^{2}-a_{k n}^{2}\right)+\sum \alpha_{k} \alpha_{r}\left(a_{k 1}^{2}-a_{r 1}^{2}+a_{r n}^{2}\right. \\
& \left.-a_{k n}^{2}\right)+2 \alpha_{1} \alpha_{n} a_{n 1}^{2}+\alpha_{n} \Sigma \alpha_{r}\left(a_{n 1}^{2}+a_{n r}^{2}-a_{1 r}^{2}\right) \text {; } \\
& r, k=2,3, \ldots(n-1) \text {, }
\end{aligned}
$$

the first and sixth terms in the right-hand member cancel, as do also the second and fourth, the third and seventh, while the fifth vanishes identically, since any two terms of the forms

$$
\alpha_{k} \alpha_{r}\left(a_{k 1}^{2}-a_{r 1}^{2}+a_{r n}^{2}-a_{k n}^{2}\right), \quad \alpha_{r} \alpha_{k}\left(a_{r 1}^{2}-a_{k 1}^{2}+a_{k_{n}}^{2}-a_{r n}^{2}\right)
$$

have the sum zero. We have therefore

$$
\sum \alpha_{k} \frac{d\left(p_{k}^{2}\right)}{d \alpha_{1}}=0 ; k=1,2, \ldots n
$$


and similarly

$$
\Sigma \alpha_{k} \frac{d\left(p_{k}^{2}\right)}{d \alpha_{r}}=0 ; k=1,2, \ldots n,
$$

if $r$ denotes any one of the numbers from 1 to $n$.

As mentioned above, $\alpha_{1}$ is the $(n-1)$ !-fold volume of the $(n-1)$-dimensional $n$-gon formed by the points $P, e_{2}, e_{3}, \ldots$ $e_{n}$; hence

$$
\alpha_{1}=p_{2} p_{3} \ldots p_{n} \sin \phi_{1},
$$

where $\phi_{1}$ denotes the $(n-1)$-dimensional angle formed by the $(n-1)$ edges $p_{2}, p_{3}, \ldots p_{n}$, the function sin $\phi_{1}$ being defined by formula (10).

Similarly we have in general

$$
\alpha_{k}=p_{1} p_{2} \cdots p_{k-1} p_{k+1} \cdots p_{n} \sin \phi_{k} .
$$

Substituting this value for $\boldsymbol{\alpha}_{\boldsymbol{k}}$ in (9), we have

$\Sigma p_{1} p_{2} \ldots p_{k-1} p_{k+1} \ldots p_{n} \sin \phi_{k} \frac{d\left(p_{k}^{2}\right)}{d \alpha_{r}}=0 ; k=1,2, \ldots n$,

or, performing the differentiation and omitting the factor $p_{1} \ldots p_{n}$ which is common to all terms,

$$
\Sigma \sin \phi_{k} \frac{d p_{k}}{d \alpha_{r}}=0 ; \quad k=1,2, \ldots n .
$$

We have thus found the general differential equation between the distances of a variable point $P$ from $n$ fixed points $e_{1}, e_{2}, \ldots e_{n}$ in $(n-1)$-dimensional space.

3. In particular we have for the plane $(n=3)$

$$
\sin \phi_{1} \frac{d p_{1}}{d \alpha_{r}}+\sin \phi_{2} \frac{d p_{2}}{d \alpha_{r}}+\sin \phi_{3} \frac{d p_{3}}{d \alpha_{r}}=0
$$

where $\phi_{1}$, for instance, is the angle between the vectors $p_{2}$ and $p_{3}$, and where $r$ may be 1 or 2 or 3 .

Similarly, for ordinary space $(n=4)$ we have:

$$
\sin \phi_{1} \frac{d p_{1}}{d \alpha_{r}}+\sin \phi_{2} \frac{d p_{2}}{d \alpha_{r}}+\sin \phi_{3} \frac{d p_{3}}{d \alpha_{r}}+\sin \phi_{4} \frac{d p_{4}}{d \alpha_{r}}=0 .
$$

Putting $\Varangle e_{r} P e_{s}=\phi_{r s}$, we have for instance

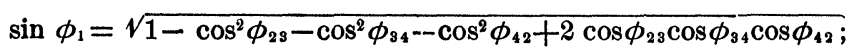


and substituting this value in the formula

$$
\alpha_{1}=p_{2} p_{3} p_{4} \sin \phi_{1},
$$

which follows from (10), we find the well-known expression for six times the volume of the tetrahedron.* The formula (15) expresses the sine of the (three-dimensional) trihedral angle by means of ordinary sine functions.

4. Minimum of the sum of the distances of the point $P$ from the points $e_{1}, e_{2}, \ldots e_{n}$. Let us now determine the minimum of the function

$$
s=\lambda_{1} p_{1}{ }^{u}+\lambda_{2} p_{2}{ }^{u}+\ldots+\lambda_{n} p_{n}{ }^{n},
$$

where $u$ is arbitrary, while $\lambda_{1}, \lambda_{2}, \ldots \lambda_{n}$ may have any positive values. We must have

or

$$
\frac{d s}{d \alpha_{1}}=\frac{d s}{d \alpha_{2}}=\ldots=\frac{d s}{d \alpha_{n}}=0,
$$

$$
\begin{gathered}
\lambda_{1} u p_{1}{ }^{u-1} \frac{d p_{1}}{d \alpha_{r}}+\lambda_{2} u p_{2}{ }^{u-1} \frac{d p_{2}}{d \alpha_{r}}+\ldots+\lambda_{n} u p_{n}{ }^{u-1} \frac{d p_{n}}{d \alpha_{r}}=0 ; \\
r=1,2, \ldots n .
\end{gathered}
$$

Now, by (12),

$$
\sin \phi_{1} \frac{d p_{1}}{d \alpha_{r}}+\sin \phi_{2} \frac{d p_{2}}{d \alpha_{r}}+\ldots+\sin \phi_{n} \frac{d p_{n}}{d \alpha_{r}}=0 ;
$$

hence we have immediately

$\lambda_{1} p_{1}{ }^{u-1}: \lambda_{2} p_{2}{ }^{u-1}: \ldots: \lambda_{n} p_{n}{ }^{u-1}=\sin \phi_{1}: \sin \phi_{2}: \ldots: \sin \phi_{n}$.

These are, in their most general form, the conditions for a minimum in our problem.

5. In particular, we have for the plane $(n=3)$

$$
\lambda_{1} p_{1}{ }^{u-1}: \lambda_{2} p_{2}{ }^{u-1}: \lambda_{3} p_{3}{ }^{u-1}=\sin \phi_{1}: \sin \phi_{2}: \sin \phi_{3},
$$

which agrees with the conditions given by Steiner for this case. $f$ Similarly we have for ordinary space $(n=4)$

$$
\begin{aligned}
\lambda_{1} p_{1}{ }^{u-1}: \lambda_{2} p_{2}{ }^{u-1}: \lambda_{3} p_{3}{ }^{{ }^{u-1}}: & \lambda_{4} p_{4}{ }^{u-1} \\
& =\sin \phi_{1}: \sin \phi_{2}: \sin \phi_{3}: \sin \phi_{4} .
\end{aligned}
$$

\footnotetext{
* This agrees with von Staudt's definition of the sine of a trihedral angle. See Crelle's Journal für Mathematik, vol. 24, p. 255, No. 8.

† Steiner, Gesam. Werke, vol. II. p. 17; Crelle's Journal für Mathematik, vol. 13, p. 362. Compare the derivation of this special result given by the author in $E l$ progreso matematico, Zaragoza, vol. 4, p. 174 ; also K. Simon, Ueber den Punkt kleinster Entfernungssumme, Dissertation, Halle, 1887 ; UHLICH, Programmabhandlung, Grimma 1886 ; DiPPE, in Crelle's Journal für Mathematik, vol. 16.
} 
With $\lambda_{1}=\lambda_{2}=\lambda_{3}=\lambda_{4}$ and $u=1$ this reduces to

$$
\sin \phi_{1}=\sin \phi_{2}=\sin \phi_{3}=\sin \phi_{4} \text {. }
$$

As these quantities, by (16), measure the trihedral angles formed at $P$ by any three of the vectors $p_{1}, p_{2}, p_{3}, p_{4}$, it appears that the condition for the minimum point (as we may call it for the sake of brevity) is the equivalence (as to volume) of these trihedral angles, or the equivalence (as to area) of the triangles cut out by the faces of these angles on a sphere described about $P$. If the radius of this sphere be taken as 1 , the area of each triangle is $\pi$.

Let the vectors $P e_{1}, P e_{2}, P e_{3}, P e_{4}$ intersect the sphere in the points $P_{1}, P_{2}, P_{3}, P_{4}$. Four such points (by means of which equivalent trihedral angles and spherical triangles can be constructed) may be found by assuming arbitrarily two points, say $P_{1}$ and $P_{2}$, drawing the diameters $P_{1} P_{3}$ and $P_{2} P_{4}$, and turning the angle $P_{3} P P_{4}$ about its bisector through any angle.* It then appears that the four trihedral angles, as well as the corresponding spherical triangles, are not merely equivalent, but equal.

For, substituting in (23) for the sines their values from (16) and the analogous formulæ, and suppressing the common factor, we find

$$
\alpha_{1} p_{1}=\alpha_{2} p_{2}=\alpha_{3} p_{3}=\alpha_{4} p_{4}
$$

i.e., we have, for instance, $\alpha_{3}: \alpha_{4}=p_{4}: p_{3}$. But as the tetrahedra $\alpha_{3}, \alpha_{4}$ have the common base $P e_{1} e_{2}$, their volumes are proportional to the perpendiculars $h_{4}, h_{3}$, let fall from $e_{4}, e_{3}$ on this base; hence

$$
\frac{p_{4}}{p_{3}}=\frac{h_{4}}{h_{3}}, \quad \text { or } \quad \frac{h_{3}}{p_{3}}=\frac{h_{4}}{p_{4}} .
$$

This means that the sines of the angles at which the vectors $p_{3}$ and $p_{4}$ are inclined to the plane $P e_{1} e_{2}$, and hence these angles themselves, are equal. It follows that the planes $p_{3} p_{4}$ and $p_{1} p_{2}$ intersect in the bisector of the angle $\left(p_{3} p_{4}\right)$. As the corresponding relation must hold for the angle $\left(p_{1} p_{2}\right)$, it appears that the bisectors of the angles $\left(p_{3} p_{4}\right)$ and $\left(p_{1} p_{2}\right)$ coincide. For a similar reason the bisectors of $\left(p_{1} p_{3}\right)$ and $\left(p_{2} p_{4}\right)$, as well as those of $\left(p_{1} p_{4}\right)$ and $\left(p_{2} p_{3}\right)$ must coincide. We have therefore

$$
\left(p_{1} p_{2}\right)=\left(p_{3} p_{4}\right), \quad\left(p_{1} p_{3}\right)=\left(p_{2} p_{4}\right), \quad\left(p_{1} p_{4}\right)=\left(p_{2} p_{3}\right) ;
$$

* Comp. Sturm, l. c. p. 57. 
and any two of these angles can, by rotation about the common bisector, be brought into the position of vertical angles, or, conversely, can be generated from this position.

6 . If one of the trihedral angles of the given tetrahedron, say $e_{4}$, has the value $\pi$, the point $P$ will coincide with $e_{4}$; the direction of $p_{4}$ becomes indeterminate since the vector $p_{4}=0$ and, by (22), sin $\phi_{4} \cdot p_{3}{ }^{u-1}=0^{0} . \sin \phi_{3}$, i.e., $\sin \phi_{4}$ is an indeterminate quantity. It follows that $P$ will coincide with $e_{4}$ not only for the value $\pi$, but for any greater value of the trihedral angle.

Thus, if the trihedral angle $e_{4}$ be $=2 \pi$, the point $e_{4}$ lies within the triangle $e_{1} e_{2} e_{3}$. Hence, if one of four points of the same plane lies within the triangle formed by the others, this point is the minimum point for the four points.

If the above-mentioned rotation of the angle $P_{3} P P_{4}$ be equal to zero, the points $e_{1}, e_{2}, e_{3}, e_{4}$ form an ordinary quadrilateral in which each of the four points lies outside the triangle of the three others; and $P$ is the intersection of the diagonals and at the same time the minimum point.

If the vertices of the quadrilateral happen to lie in a straight line, say in the order $e_{1}, e_{2}, e_{3}, e_{4}$, then the diagonals $e_{1} e_{3}$ and $e_{2} e_{4}$ have in common the vector $e_{2} e_{3}$, instead of a point of intersection, and each point of this vector has the minimum property.

\%. With $\lambda_{1}=\lambda_{2}=\lambda_{3}=\lambda_{4}$ and $u=2$, (22) reduces to

$$
p_{1}: p_{2}: p_{3}: p_{4}=\sin \phi_{1}: \sin \phi_{2}: \sin \phi_{3}: \sin \phi_{4},
$$

or, by (16),

$$
p_{1}: p_{2}: p_{3}: p_{4}=\frac{\alpha_{1}}{2 p_{2} p_{3} p_{4}}: \frac{\alpha_{2}}{2 p_{3} p_{4} p_{1}}: \frac{\alpha_{3}}{2 p_{4} p_{1} p_{2}}: \frac{\alpha_{4}}{2 p_{1} p_{2} p_{3}},
$$

or, finally,

$$
\alpha_{1}=\alpha_{2}=\alpha_{3}=\alpha_{4}
$$

that is, the planes passing through $P$ and the edges of the tetrahedron divide the volume of the tetrahedron into four equal parts. Equation (1) gives in this case

$$
P=\frac{e_{1}+e_{2}+e_{3}+e_{4}}{4} \text {. }
$$

Either of these properties characterizes the point $P$ as the centroid (barycentre, centre of gravity) of the tetrahedron.*

* Glaser (Programm, Homburg v. d. H., 1887) finds as minimum point the intersection of the lines joining the mid-points of any two opposite edges; this is again the centroid. For a simple proof of this 
It retains this property if the four points $e_{1}, e_{2}, e_{3}, e_{4}$ lie in the same plane or in the same line.

This result can evidently be extended at once to the case of any $n$ points in a space of $n-1$ dimensions. If the squares of the distances are multiplied by factors $\lambda_{1}, \lambda_{2}, \lambda_{3}$, $\lambda_{4}$, it is only necessary, in determining the centroid, to give the points these weights.*

8. Geometrical interpretations of the general result.-For the purpose of geometrical interpretation it will be well to remove the sine-functions from formula (20). This will also make it possible to extend the problem to groups of $n$ points situated in a space of less than $(n-1)$ dimensions; the sines in this case would all vanish.

According to Grassmann's geometrical calculus the outer product of two vectors $p_{2}$ and $p_{3}$, including the angle $\phi_{1}$, is

$$
\left(p_{2} p_{3}\right)=p_{2} p_{3} \sin \phi_{1} \text {. }
$$

Both members of this equation express also the area $\left(\alpha_{1}\right)$ of the parallelogram deternined by the three parts $p_{2}, p_{3}, \phi_{1}$.

Similarly, in three-dimensional space, we have for three vectors $p_{2}, p_{3}, p_{4}$, forming the trihedral angle $\phi_{1}$,

$$
\alpha_{1}=\left(p_{2} p_{3} p_{4}\right)=2 p_{2} p_{3} p_{4} \sin \phi_{1}
$$

as the volume of the parallelepiped determined by the four parts $p_{2}, p_{3}, p_{4}, \phi_{1}$ (comp. formula (16)).

In general we have in an $(n-1)$-dimensional region (comp. formula (10))

$$
\alpha_{1}=\left(p_{2} p_{3} \ldots p_{n}\right)=(n-2) ! p_{2} p_{3} \ldots p_{n} \sin \phi_{1}
$$

as the $(n-1)$-dimensional volume of the configuration determined by the edges $p_{2}, p_{3}, \ldots p_{n}$ and the $(n-1)$-dimensional angle $\phi_{1}$.

Similarly,

$$
\begin{aligned}
\alpha_{k} & =\left(p_{1} p_{2} \ldots p_{k-1} p_{k+1} \cdots p_{n}\right) \\
& =(n-2) ! p_{1} p_{2} \cdots p_{k-1} p_{k+1} \cdots p_{n} \sin \phi_{k}
\end{aligned}
$$

proposition by meaus of the geometrical calculus see the author's paper Quelques théorèmes de géométrie à $n$ dimensions, in Bull. de la Soc. math. de France, vol. 10, p. 176.

* This general problem will be found solved in Grassmann's Aus. dehnungslehre, 1862, p. 215. 
Hence

$$
\sin \phi_{k}=\frac{\left(p_{1} \ldots p_{k-1} p_{k+1} \cdots p_{n}\right)}{(n-2) ! p_{1} \ldots p_{k-1} p_{k+1} \cdots p_{n}}
$$

With the aid of this formula we find from (20), for instance,

or

$$
\begin{aligned}
\frac{\lambda_{1} p_{1}{ }^{u-1}}{\lambda_{2} p_{2}{ }^{u-1}} & =\frac{\left(p_{2} p_{3} \cdots p_{n}\right)}{(n-2) ! p_{2} p_{3} \cdots p_{n}}: \frac{p_{1} p_{3} \cdots p_{n}}{(n-2) ! p_{1} p_{3} \cdots p_{n}} \\
& =\frac{\left(p_{2} p_{3} \cdots p_{n}\right)}{\left(p_{1} p_{3} \cdots p_{n}\right)} \cdot \frac{p_{1}}{p_{2}}
\end{aligned}
$$

$$
\frac{\lambda_{1} p_{1}{ }^{u-2}}{\lambda_{2} p_{2}{ }^{u-2}}=\frac{\left(p_{2} p_{3} \cdots p_{n}\right)}{\left(p_{1} p_{3} \cdots p_{n}\right)}
$$

In general, we have

$$
\begin{aligned}
\frac{\lambda_{r} p_{r}{ }^{\mu-2}}{\lambda_{s} p_{s}{ }^{u-2}} & =\frac{\left(p_{1} \ldots p_{r-1} p_{r+1} \cdots p_{n}\right)}{\left(p_{1} \cdots p_{s-1} p_{s+1} \cdots p_{n}\right)} \\
r, s & =1,2, \ldots n ; r \gtrless s .
\end{aligned}
$$

Thus the sine-functions in (20) are replaced by $(n-1)$-dimensional volumes.

9 . The formula just derived is not directly applicable to $n$ points in spaces of less than $(n-1)$ dimensions. But it is closely related to a formula suited for this purpose; and this formula we now proceed to develop.

Let $A_{r}$ be one of the $n$ fixed points, $P$ a variable point, and $O$ an arbitrary fixed point. We put

$(O-P)=(\rho), \quad\left(O-A_{r}\right)=\left(\rho_{r}\right), \quad\left(A_{r}-P\right)=\left(p_{r}\right)$,

where the parentheses indicate that the quantities enclosed are vectors, i.e., have not merely length but also direction. By the ordinary laws of vector analysis we have

$$
\left(O-A_{r}\right)+\left(A_{r}-P\right)=(O-P), \text { or } \quad\left(\rho_{r}\right)+\left(p_{r}\right)=(\rho) \text {, }
$$

or

$$
\left(p_{r}\right)=(\rho)-\left(\rho_{r}\right) \text {. }
$$

If $p_{r}$ be the numerical value of $\left(p_{r}\right)$, we have

or

$$
p_{r}=+\sqrt{\left(p_{r}\right)^{2}},
$$

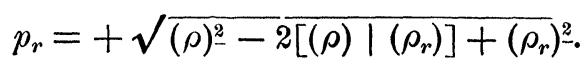


Hence

$$
\begin{array}{r}
\frac{d p_{r}}{d \rho}=\frac{1}{p_{r}}\left[(\rho)-\frac{\left[(\rho) \mid\left(\rho_{r}\right)\right]}{\rho}\right]=\frac{[(\rho) \mid(\rho)]-\left[(\rho) \mid\left(\rho_{r}\right)\right]}{p_{r} \rho} \underline{ } \\
=\frac{\left((\rho) \mid\left[(\rho)-\left(\rho_{r}\right)\right]\right)}{p_{r} \rho}=\frac{\left[(\rho) \mid\left(p_{r}\right)\right]}{\rho p_{r}},
\end{array}
$$

or if we omit the parentheses, since in the inner product vectors only can be used,

$$
\frac{d p_{r}}{d \rho}=\frac{\left(\rho \mid p_{r}\right)}{\rho p_{r}}=\frac{\left(p_{r} \mid \rho\right)}{p_{r} \rho}
$$

Now, to make the expression

$$
s=\lambda_{1} p_{1}{ }^{u}+\lambda_{2} p_{2}{ }^{u}+\ldots+\lambda_{n} p_{n}{ }^{u}
$$

a minimum, we must have

or

$$
\Sigma \lambda_{r} p_{r}{ }^{u-1} \frac{d p_{r}}{d \rho}=0 ; \quad r=1,2, \ldots n
$$

$\lambda_{1} p_{1}{ }^{u-1} \frac{\left(p_{1} \mid \rho\right)}{p_{1} \rho}+\lambda_{2} p_{2}{ }^{u-1} \frac{\left(p_{2} \mid \rho\right)}{p_{2} \rho}+\ldots+\lambda_{n} p_{n}{ }^{u-1} \frac{\left(p_{n} \mid \rho\right)}{p_{n} \rho}=0$

or, finally,

$$
\left(\left[\lambda_{1} p_{1}{ }^{u-1} \frac{\left(p_{1}\right)}{p}+\lambda_{2} p_{2}{ }^{u-1} \frac{\left(p_{2}\right)}{p_{2}}+\ldots+\lambda_{n} p_{n}{ }^{u-1} \frac{\left(p_{n}\right)}{p_{n}}\right] \mid \rho\right)=0 .
$$

The meaning of this equation is that the vector enclosed in the square brackets is perpendicular to the vector $\rho$ which joins the arbitrary point $O$ to the minimum point $P$. As the direction of $\rho$ is arbitrary, equation (33) can evidently hold only if the vector that forms the first factor of the inner product has the value 0 . Hence the minimum condition is

$$
\lambda_{1} p_{1}{ }^{u-1} \frac{\left(p_{1}\right)}{p_{1}}+\lambda_{2} p_{2}{ }^{u-1} \frac{\left(p_{2}\right)}{p_{2}}+\ldots+\lambda_{n} p_{n}{ }^{u-1} \frac{\left(p_{n}\right)}{p_{n}}=0 .
$$

Considering that $\frac{\left(p_{r}\right)}{p_{r}}$ is a unit vector parallel to $\left(p_{r}\right)$, we find our equation to have the following meaning: On the 
lines $P A_{1}, P A_{2}, \ldots P A_{n}$ lay off unit lengths from the minimum point $P$ as origin, increase these lengths in the ratios $1: \lambda_{1} p_{1}{ }^{u-1}, 1: \lambda_{2} p_{2}{ }^{u-1}, \ldots 1: \lambda_{n} p_{n}{ }^{u-1}$, and displace them parallel to themselves so that the initial point of each coincides with the terminal point of the one preceding. The result of this construction will be a closed polygon.

This condition for the minimum point is evidently independent of the number of dimensions of the space in which the points $A_{1}, A_{2}, \ldots A_{n}$ are given. Moreover, as every vector $p$ occurring in the construction has a definite direction, relative minima, such as might arise when one of the terms of the sum should become negative, are excluded.

It should also be noticed that the present method is entirely independent of the preceding investigations. Nevertheless it is easy to transform formula (34) so as to reduce to (30), and thus to establish the required connection between the two methods.

10. Removing the denominators in (34), we find

$$
\begin{aligned}
\lambda_{1} p_{1}{ }^{n-1} p_{2} p_{3} \cdots p_{n}\left(p_{1}\right)+\lambda_{2} p_{2}{ }^{u-1} p_{3} & \cdots p_{n} p_{1}\left(p_{2}\right)+\cdots \\
& +\lambda_{n} p_{n}{ }^{u-1} p_{1} p_{2} \cdots p_{n-1}\left(p_{n}\right)=0 .
\end{aligned}
$$

If this equation be multiplied by the outer product

$$
\left(p_{1} p_{2} \cdots p_{r-1} p_{r+1} \cdots p_{s-1} p_{s+1} \cdots p_{n}\right),
$$

all terms excepting those that contain $\left(p_{r}\right)$ and $\left(p_{s}\right)$ will vanish so as to leave

$$
\begin{aligned}
& \lambda_{r} p_{r}{ }^{u-1} p_{1} p_{2} \cdot p_{r-1} p_{r+1} \cdots p_{n}\left(p_{r} p_{1} \ldots p_{r-1} p_{r+1} \cdots p_{s-1} p_{s+1} \cdots p_{n}\right) \\
& \quad+\lambda_{s} p_{s}{ }^{u-1} p_{1} p_{2} \cdots p_{s-1} p_{s+1} \cdots p_{n}\left(p_{s} p_{1} \ldots p_{r-1} p_{r+1} \cdots p_{s-1} p_{s+1} \cdots p_{n}\right)=0
\end{aligned}
$$

or, omitting the numerical factors common to both terms,

$$
\begin{aligned}
\lambda_{r} p_{r}{ }^{u-1} p_{s}\left(p_{1} p_{2} \cdots p_{s-1} p_{s+1} \cdots p_{n}\right) & \\
& = \pm \lambda_{s} p_{s}{ }^{u-1} p_{r}\left(p_{1} p_{2} \cdots p_{r-1} p_{r+1} \cdots p_{n}\right)=0 .
\end{aligned}
$$

The double sign is due to the fact that the introduction of the factors $p_{r}$ and $p_{s}$ into their natural places may require an equal or unequal number of changes of sign. As, however, equation (30) is merely a relation between absolute values, we may drop the double sign; the last equation then reduces to the following:

$$
\frac{\lambda_{r} p_{r}{ }^{u-2}}{\bar{\lambda}_{s} p_{s}^{u-2}}=\frac{\left(p_{1} \ldots p_{r-1} p_{r+1} \cdots p_{n}\right)}{\left(p_{1} \cdots p_{s-1} p_{s+1} \cdots p_{n}\right)}
$$

which agrees with (30). 
Now (30) was obtained as merely another form of (20); hence we have thus also established the connection between the $n$-dimensional generalizations of the solutions formulated by Steiner and Sturm.

11. If one of the given points, say $A_{n}$, is to be the minimum point, then $\left(p_{n}\right)=p_{n}=0$, hence $\frac{\left(p_{n}\right)}{p_{n}}$ is indeterminate; and owing to the factor $p_{n}{ }^{u-1}$ the last term in (34) drops out provided that $u>1$. If, however, $u=1$, the factor $p_{n}{ }^{u-1}$, and hence the whole last term, is indeterminate; that is, the closing side of the polygon has no determinate value. But we can find a limit for it by the following consideration. According to the laws of the geometrical calculus we have

$$
\frac{\left(p_{r} \mid \rho\right)}{p_{r} \rho}=\cos \left(p_{r} \rho\right) \text {. }
$$

Taking the form (32a) for the minimum condition, we notice first that in the triangle $A_{n} P O$ the angle $P O A_{n}=0$ if $P$ coincides with $A_{n}$. The sum of the other two angles is $\pi$, while either of these angles, taken separately, is indeterminate. Hence $\Varangle A_{n} P O$, or $\Varangle\left(p_{n} \rho\right)$, and consequently $\cos \left(p_{n} \rho\right)$, is indeterminate. By comparing equations (32a) and (34) it will be seen that this cosine has the same value as the closing side of the polygon, which must therefore be less than (or equal to) the unit of length.*

12. With $u=1$ and $\lambda_{1}=\lambda_{2}=\ldots=\lambda_{n}=1$, (34) assumes the simple form

$$
\frac{\left(p_{1}\right)}{p_{1}}+\frac{\left(p_{2}\right)}{p_{2}}+\ldots+\frac{\left(p_{n}\right)}{p_{n}}=0
$$

which agrees with the result found by Sturm, $\nmid$ that the vectors $\left(p_{1}\right),\left(p_{2}\right), \ldots\left(p_{n}\right)$ agree in direction with the sides of a closed polygon all of whose sides have the length 1 .

With $u=2$, (34) becomes

$$
\lambda_{1}\left(p_{1}\right)+\lambda_{2}\left(p_{2}\right)+\ldots+\lambda_{n}\left(p_{n}\right)=0 ;
$$

or if the value $\left(A_{r}-P\right)$ be substituted for $p_{r}$ from (31):

$$
P=\frac{\lambda_{1} A_{1}+\lambda_{2} A_{2}+\ldots+\lambda_{n} A_{n}}{\lambda_{2}+\lambda_{1}+\ldots+\lambda_{n}}
$$

* This agrees with STuRM, l.c. p. 52. This special case is designated by Sturm (p. 58) as an Umartung of the minimum figure.

+ L. c. p. 54 , No. 5 . 
that is, $P$ is the centroid of the points $A_{1}, A_{2}, \ldots A_{n}$, these points having the weights $\lambda_{1}, \lambda_{2}, \ldots \lambda_{n}$. (Comp. Art. $\%$, end.)

13. Substituting the same value of $p_{r}$ in (34), we find

$$
P=\frac{\lambda_{1} p_{1}{ }^{u-2} A_{1}+\lambda_{2} p_{2}{ }^{u-2} A_{2}+\ldots+\lambda_{n} p_{n}{ }^{u-2} A_{n}}{\lambda_{1} p_{1}{ }^{u-2}+\lambda_{2} p_{2}{ }^{u-2}+\ldots+\lambda_{n} p_{n}{ }^{u-2}}
$$

that is: In a space of any number of dimensions, let $p_{1}, p_{2}$, $\ldots p_{n}$ be the distances of a variable point $P$ from $n$ fixed points $A_{1}, A_{2}, \ldots A_{n}$; then the function $s=\lambda_{1} p_{1}{ }^{2}+\lambda_{2} p_{2}{ }^{u}$ $+\ldots+\lambda_{n} p_{n}{ }^{\prime}$ has its minimum value when $P$ is taken as the centroid of the points $A_{1}, A_{2}, \ldots A_{n}$, the weights given to these points being $\lambda_{1} p_{1}{ }^{u-2}, \lambda_{2} p_{2}{ }^{u-2}, \ldots \lambda_{n} p_{n}{ }^{u-2}$.

The minimum point has thus been found as a centroid in the most general case.

In particular, with $\iota=1, n=3$, and $\lambda_{1}=\lambda_{2}=\lambda_{3}=1$, we have the corollary: Let $p_{1}, p_{2}, p_{3}$ be the distances of the vertices of a triangle from the point $P$ for which the sum of the distances is a minimum; then $P$ is the centroid of the vertices, the weights of these points being $\frac{1}{p_{1}}, \frac{1}{p_{2}}, \frac{1}{p_{3}}$.

It further appears from (39) that $P$ can be constructed as centroid only in the case $u=2$, since in all other cases the weights to be given to the points $A_{1}, A_{2}, \ldots A_{n}$ are functions of the unknown distances $p_{1}, p_{2}, \ldots p_{n}$.

14. Remarks concerning the construction of the minimum point.-In the case $u=2$ the construction of the minimum point reduces, by formula (39), to an ordinary construction of a centroid because the unknown quantities $p_{1}, p_{2}, \ldots p_{n}$ disappear from the right-hand member.

In the case $u=1$, formula (20) is simplified in a similar way and shows that the sines of the $(n-1)$-dimensional angles formed by joining $P$ to the $n$ given points are in the ratio of the given numbers $\lambda_{1}, \lambda_{2}, \ldots \lambda_{n}$. If, in particular, these numbers are all equal, the corresponding angles are equal, as was known to be the case for $n=3$ and $n=4$. To construct the point it is only necessary to satisfy this condition; this problem seems to have been solved only for $n=3$.

But formula (20), as well as (34), can be used with any value of the numbers $u, \lambda_{1}, \lambda_{2}, \ldots \lambda_{n}$ for the purpose of solving the converse problem : a point $P$ being given in an $(n-1)$-dimensional space, to find a system of $n$ points, for which $P$ is the minimum point satisfying the condition (17). For, let $c$ be any constant vector, subject only to the condition 
$c>\lambda_{r} p_{r}{ }^{\mu-1}$ for every one of the values $r=1,2, \ldots n$, and let us put, conformably to (20),

$$
\frac{\lambda_{r} p_{r}{ }^{n-1}}{\sin \phi_{r}}=c, \quad \text { and hence } \sin \phi_{r}=\frac{\lambda_{r} p_{r}{ }^{u-1}}{c} ;
$$

then the following construction results for the required points. From $P$ draw any $n$ half-rays such that no $(n-s)$ rays lie in a space of less than $(n-s)$ dimensions ; these rays fully determine the $n$ quantities $\sin \phi_{r}$. Every point $A_{r}$ of the required system is now given on its half-ray by means of its distance $p_{r}$ from $P$ which by (40) becomes

$$
p_{r}=\sqrt[u-1]{\frac{c \sin \phi_{r}}{\lambda_{r}}} .
$$

If $u=1$, the points may have any position on their half-rays.

After thus deducing from (20) the construction, in special cases, of the minimum point for $n$ points in an $(n-1)$-dimensional space, it remains to show how these results can be extended to any number of points in such a space.

15. This generalization can be effected by first allowing one of the $n$ points to be removed to the $(n-2)$-dimensional space determined by the $(n-1)$ remaining points, or by projecting the whole group of points on the $(n-2)$-dimensional space, and by determining the effect of this transformation on the position of the minimum point. Next, 2 points of the new group are removed to the $(n-3)$-dimensional space determined by the $(n-2)$ remaining points; and so on. In this way any number of points in a space of any number of dimensions can be investigated.

The groups of points obtained in this way will show interesting spatial relations which affect the position of the minimum point. ${ }^{*}$ The simplest cases may serve for illustration.

(1) Let $P$ be the point the sum of whose distances from three points in a plane $A_{1}, A_{2}, A_{3}$ is a minimum. As $A_{3}$ approaches the straight line $A_{1} A_{2}$, the point $P$ will approach $A_{3}$, coinciding with it as soon as $\Varangle A_{1} A_{3} A_{2}=120^{\circ}$; and $A_{3}$ henceforth remains minimum point even when $A_{3}$ comes to lie on the vector $A_{1} A_{2}$. - If $A_{3}$ approaches the prolongation of this vector, say on the side of $A_{1}$, the point $A_{1}$ becomes minimum point as soon as $\nvdash A_{3} A_{1} A_{2}=120^{\circ}$, and remains so even

\footnotetext{
* As to these relations see the paper by the author: Ueber die verschiedenen Formen von Gruppen, welche $r$ beliebige Punkte im n-dimensionalen Raume bilden können, in Hoppe's Archiv der Math. und Phys. (2), vol. 10, (1891), p. 292 sq.
} 
when $A_{3}$ comes to lie on $A_{2} A_{1}$ produced. Hence, for three points in a line, the one included between the two others is always the minimum point.

(2) Let $P$ be the point for which the sum of the distances from four points in space $A_{1}, A_{2}, A_{3}, A_{4}$ is a minimum. These points can come to fall into a plane in two different ways: First, it is possible that one of the points, $A_{4}$, approaches the area of the triangle $A_{1} A_{2} A_{3}$. Then $P$ will come to coincide with $A_{4}$ as soon as the trihedral angle whose vertex is $A_{4}$ has become equal to $\pi$, and $A_{4}$ remains minimum point even when this point falls into the area $A_{1} A_{2} A_{3}$. - Secondly, the edge $A_{1} A_{2}$ may move until it comes to intersect $A_{3} A_{4}$. As the angles $A_{1} P A_{2}$ and $A_{3} P A_{4}$, as well as the angles $A_{1} P A_{4}$ and $A_{2} P A_{3}$, must have the same bisectors, the point $P$ will now come to coincide with the intersection of the diagonals of the quadrilateral $A_{1} A_{2} A_{3} A_{4}$.

If, moreover, in the former of these two cases the points $A_{1}$, $A_{2}, A_{3}$ fall into a straight line, while the minimum point $A_{4}$ is constantly situated within the triangle $A_{1} A_{2} A_{3}$, the point $A_{4}$ itself will fall into this line. But now $A_{4}$ is no more the only minimum point; every point of the vector $A_{2} A_{4}$ will have this property. In the latter of the above cases, if by turning one diagonal until it coincides with the other, the four points be brought into the same straight line, every point of the shorter diagonal, being a point common to both diagonals, is a minimum point, conformably to the preceding result. (Compare also Art. 6.)

16. The construction of the system $A_{1}, A_{2}, \ldots A_{n}$ for a given minimum point $P$ is, however, effected far more readily by means of the more general formula (34). It is only necessary to construct in $(n-1)$-dimensional space a closed polygon having $n$, or any greater number of, equal sides, to transfer these sides to parallel positions with the common origin $P$, and to stretch these vectors in the ratios $1: \lambda_{1} p_{1}{ }^{u-1}$, $1: \lambda_{2} p_{2}{ }^{u-1}, \ldots 1: \lambda_{n} p_{n}{ }^{u-1}$. The terminal points of the stretched vectors are the required points.

1\%. The construction of the point $P$ from given points $A_{1}$, $A_{2}, \ldots A_{n}$ can be reduced to the solution of an algebraic equation when the points are situated on a straight line.

Let the straight line be determined by the points $e_{1}$ and $e_{2}$, and let

$$
P=\alpha e_{1}+(1-\alpha) e_{2} ; \quad A_{r}=\alpha_{r} e_{1}+\left(1-\alpha_{r}\right) e_{2}
$$

Then we have

$$
\left(p_{r}\right)=\left(A_{r}-P\right)=\left(\alpha-\alpha_{r}\right)\left(e_{1}-e_{2}\right),
$$


or, denoting the unit of length $\left(e_{1}-e_{2}\right)$ by $a_{r}$,

$$
\left(p_{r}\right)=\left(\alpha-\alpha_{r}\right)\left(a_{r}\right) ; p_{r}=\alpha-\alpha_{r},
$$

whence

$$
\frac{\left(p_{r}\right)}{p_{r}}=\left(a_{r}\right)
$$

By the substitution of this value the condition (34) becomes

$$
\lambda_{1} p_{1}{ }^{u-1}\left(a_{1}\right)+\lambda_{2} p_{2}{ }^{u-1}\left(a_{2}\right)+\ldots+\lambda_{n} p_{n}{ }^{u-1}\left(a_{n}\right)=0,
$$

or, since the collinear vectors $\left(a_{1}\right),\left(a_{2}\right), \ldots\left(a_{n}\right)$ can be replaced by their numerical value $\pm a$,

$$
\pm \lambda_{1} p_{1}{ }^{u-1} a \pm \lambda_{2} p_{2}{ }^{u-1} a \pm \ldots \pm \lambda_{n} p_{n}{ }^{n-1} a=0 .
$$

Two cases must now be distinguished. (1) If $u$ be even, the powers $p_{r}{ }^{u}$ in $(1 \%)$ are positive, whether $p_{r}$ be positive or negative. The same must hold for the derivatives of these powers so that formula (44) can be written

$$
\lambda_{1} p_{1}{ }^{u-1}+\lambda_{2} p_{2}{ }^{u-1}+\ldots+\lambda_{n} p_{n}{ }^{u-1}=0,
$$

or

$\lambda_{1}\left(\alpha-\alpha_{1}\right)^{u-1}+\lambda_{2}\left(\alpha-\alpha_{2}\right)^{u-1}+\ldots+\lambda_{n}\left(\alpha-\alpha_{n}\right)^{u-1}=0$.

This equation determines the number $\alpha$ on which the position of the point $P$ depends. Thus, for $u=2$, we find

$$
\alpha=\frac{\lambda_{1} \alpha_{1}+\lambda_{2} \alpha_{2}+\ldots+\lambda_{n} \alpha_{n}}{\lambda_{1}+\lambda_{2}+\ldots+\lambda_{n}}
$$

that is, $P$ is the centroid of the points $A_{r}$, the weights being $\lambda_{r}$, as was shown above.

(2) If $u$ be odd, we must first determine the interval within which the minimum point is comprised. To do this we let $P$ coincide successively with $A_{1}, A_{2}, \ldots A_{n}$ and determine the value of $s$ in each case. Suppose this value diminishes up to $A_{r}$ and then increases; by moving the point $P$ to each side of $A_{r}$ it will appear whether the minimum point lies on $A_{r-1} A_{r}$ or on $A_{r} A_{r+1}$. If, for instance, the latter be the case, $\alpha$ must be found from the equation

$$
\begin{gathered}
\lambda_{1}\left(\alpha-\alpha_{1}\right)^{u-1}+\ldots+\lambda_{r}\left(\alpha-\alpha_{r}\right)^{u-1}-\lambda_{r+1}\left(\alpha-\alpha_{r+1}\right)^{u-1} \\
-\ldots-\lambda_{n}\left(\alpha-\alpha_{n}\right)^{u-1}=0 .
\end{gathered}
$$


18. With $u=1$ this equation assumes the form

$$
\lambda_{1}+\ldots+\lambda_{r}-\lambda_{r+1}-\ldots-\lambda_{n}=0 \text {. }
$$

It may happen that the given values of $\lambda$ satisfy this equation identically. In this case, $P$ is any point of the vector $A_{r} A_{r+1}$. If, in particular, $\lambda_{1}=\lambda_{2}=\ldots=\lambda_{n}=1$, the equation (48) is satisfied if $n$ be even and $r=\frac{1}{2} n$. Every point of the mid-vector $A_{\frac{n}{2}} A_{\frac{n}{2}+1}$ is then a minimum point.

If the quantities $\lambda$ do not satisfy (48), it becomes necessary to fall back on (43a), which now assumes the form

$$
\lambda_{1}\left(a_{1}\right)+\lambda_{2}\left(\alpha_{2}\right)+\ldots+\lambda_{n}\left(\alpha_{n}\right)=0 .
$$

This equation can be satisfied, independently of the quantities $\lambda$, if any one of the quantities $(a)$, say $\left(a_{r}\right)$, assumes the indeterminate form $\frac{0}{0}$; by (43), this requires $p_{r}=0$. Hence, in this case, one of the given points, $A_{r}$, is minimum point. If, moreover, $\lambda_{1}=\lambda_{2}=\ldots=\lambda_{n}=1$, and $n$ is odd, we have $r=\frac{1}{2}(n+1)$ and the mid-point of the $n$ points is minimum point. Simple geometrical considerations will confirm these particular results, which have also been mentioned by Mr. Sturm. They are here pointed out in order to call attention to the uniformity of our method (comp. Art. 11).

19. The most general case in which the minimum point becomes a vector is given, according to (39), by the equation

$$
\lambda_{1} p_{1}{ }^{u-2}+\lambda_{2} p_{2}{ }^{u-2}+\ldots+\lambda_{n} p_{n}{ }^{u-2}=0 \text {. }
$$

For, in this case, the centroid determined by this equation has the coefficient zero and reduces therefore to a vector, according to the rules of the geometrical calculus.

20 . 'The case $u=1$ admits a practical application. Let the points $A_{1}, A_{2}, \ldots A_{n}$ be railway stations, arbitrarily distributed along a railway line which need not be straight; and let $\lambda_{1}, \lambda_{2}, \ldots \lambda_{n}$ be numbers measuring their amount of traffic with a central station (for instance, the numbers indicating the population, or any other quantities proportional to the passenger or freight traffic with the central station); then the minimum point $P$ determines that position of the central station for which the total mutual traffic with this central station can be carried on with the least expense of money and time. An opinion can thus be formed as to the best position for erecting a new central station, or as to the relative advantages of one already in existence.*

* On the extension of the minimum problem to a group of points which continuously fill a vector, an area, or a volume, and on its appli- 
21. Application to a problem in mechanics.-Let $n$ fixed masses $\lambda_{1}, \lambda_{2}, \ldots \lambda_{n}$ be given in an $(n-1)$-dimensional space; and let these masses attract, according to Newton's law, a movable mass $\mu$, situated within the $n$-gon formed by the masses $\lambda$. Where should the mass $\mu$ be placed to make the absolute sum of the attractions exerted on it a minimum?

Let $e_{1}, e_{2}, \ldots e_{n}$ be the centroids at which the masses $\lambda$ are assumed to be concentrated, and let $P$ be determined by the equation (1), as in Art. 2. To satisfy the condition concerning the movability of $P$ it is only necessary to assume $\alpha_{1}, \alpha_{2}$, $\ldots \alpha_{n}$ all positive. Putting $u=-2$ in the equation of condition (17) and multiplying this equation by $\mu$, we find

$$
s \mu=\frac{\lambda_{1} \mu}{p_{1}^{2}}+\frac{\lambda_{2} \mu}{p_{2}^{2}}+\ldots+\frac{\lambda_{n} \mu}{p_{n}^{2}}
$$

as the function that is to become a minimum. Everything else follows by putting $u=-2$ in the results obtained before.

Special Cases. (1) $n=2$. - In this case, which on account of its simplicity was not mentioned above, we find from (30), by putting $r=1, s=2$, and from (28), with $\alpha_{1}=p_{2}, \alpha_{2}=p_{1}$,

$$
\frac{\alpha_{1}}{\alpha_{2}}=\frac{\sqrt[8]{\bar{\lambda}_{2}}}{\sqrt[3]{\lambda_{1}}}
$$

that is, the distances of the point of least attraction from the attracting points are to each other as the cube roots of the masses of these points. For it follows from the general equation of the point $P$ (comp. formula (1)), viz.,

that

$$
\left(\alpha_{1}+\alpha_{2}\right) P=\alpha_{1} e_{1}+\alpha_{2} e_{2},
$$

$$
\frac{\alpha_{1}}{\alpha_{2}}=\frac{\left(e_{2}-P\right)}{\left(P-e_{1}\right)}
$$

(2) $n=3$.-Formula (20) gives the conditions

or

$$
\lambda_{1} p_{1}^{-3}: \lambda_{2} p_{2}{ }^{-3}: \lambda_{3} p_{3}{ }^{-3}=\sin \phi_{1}: \sin \phi_{2}: \sin \phi_{3},
$$

$$
p_{1}: p_{2}: p_{3}=\sqrt[3]{\frac{\lambda_{1}}{\sin \phi_{1}}}: \sqrt[3]{\frac{\lambda_{2}}{\sin \phi_{2}}}: \sqrt[3]{\frac{\lambda_{3}}{\sin \phi_{3}}},
$$

cation to the determination of the most advantageous position of the farmhouses on a farm of given shape and area, see the paper by the author Sur les distances moyennes entre un point et des variétés de points, discrètes ou continues, in Comptes rendus de l'Association frangaise pour l'avancement des sciences, 1887, p. 266, sq., especially p. 275. 
or, if the sine functions be eliminated by means of (11),

$$
\alpha_{1}: \alpha_{2}: \alpha_{3}=\lambda_{1}\left(p_{2} p_{3}\right)^{4}: \lambda_{2}\left(p_{3} p_{1}\right)^{4}: \lambda_{3}\left(p_{1} p_{2}\right)^{4} \text {. }
$$

While (52) does not enable us to construct the point of least attraction, it furnishes a solution of the converse problem: to determine the ratios of the masses of three points so as to make the sum of their attractions on a point $P$ within their triangle a minimum.

If, in (50), we put $n=2$ and $\alpha_{1}+\alpha_{2}=1$, and hence $p_{1}+$ $p_{2}=1$, this equation can be regarded as that of a curve whose ordinate $s$ represents the sum of the attractions exerted by the points $e_{1}$ and $e_{2}$ on the foot of the ordinate. This curve approaches asymptotically the perpendiculars erected on the vector $\left(e_{1}-e_{2}\right)$ at $e_{1}$ and $e_{2}$; and the point of minimum attraction corresponds to its lowest point. Similarly, in the case $n=3$, the sum of the attractions exerted by the vertices of the triangle on any point within this triangle can be represented by the ordinate of a surface, erected at this point at right angles to the plane of the triangle. This suggestion may here suffice.

22. Concluding remark.-Further results concerning generalizations of the problem of the minimum sum of distances are reserved for a future communication.

\section{WAS THE BINOMIAL THEOREM ENGRAVEN ON NEWTON'S MONUMENT?}

BY PROFESSOR FLORIAN CAJORI.

Moritz Cantor, in a recently published part of his admirable work, Vorlesungen über Geschichte der Mathematik, speaks of the "Binomialreihe, welcher man $172 \%$ bei Newtons Tode... eine solche Wichtigkeit beilegte, dass man sie aus allen anderen Leistungen des Vestorbenen auswählte, um als Inschrift auf seinen Grabstein in der Westminsterabtei eingemeisselt zu werden" (vol. III, p. 65). In my own brief History of Mathematics, p. 218, I say that it is not true that the binomial theorem is engraved on Newton's monument.

The above passage in Cantor's work leads us to re-examine the subject The first step naturally is to question the monument, but we do so in vain. Says Dr. Granville, the present Dean of Westminster, in a letter to the writer: "In front of the half-recumbent figure of Sir Isaac Newton are two winged youths holding a small scroll in which are still, according to Neale, some mathematical figures. . . . I fear that the figures on the small marble scroll are quite obliterated. A mathemat- 